\title{
Anomalous polarization dependence in vibrationally resolved resonant inelastic X-ray scattering of $\mathrm{H}_{2} \mathrm{O}$
}

\author{
Vinícius Vaz da Cruz,,${ }^{1 *}$ Emelie Ertan, ${ }^{2}$ Nina Ignatova,,${ }^{1,3}$ Rafael C. Couto, ${ }^{1}$ Sergey \\ Polyutov ${ }^{3}$ Michael Odelius, ${ }^{2}$ Victor Kimberg, ${ }^{1,3}$ and Faris Gel'mukhanov ${ }^{1,3, \dagger}$ \\ ${ }^{1}$ Theoretical Chemistry and Biology, Royal Institute of Technology, 10691 Stockholm, Sweden \\ ${ }^{2}$ Department of Physics, Stockholm University, AlbaNova University Center, 10691 Stockholm, Sweden \\ ${ }^{3}$ Institute of Nanotechnology, Spectroscopy and Quantum Chemistry, \\ Siberian Federal University, 660041 Krasnoyarsk, Russia
}

\begin{abstract}
It is well established that different electronic channels, in resonant inelastic X-ray scattering (RIXS), display different polarization dependences due to different orientations of their corresponding transition dipole moments in the molecular frame. However, this effect does not influence the vibrational progression in the Franck-Condon approximation. We have found that the transition dipole moments of core-excitation and de-excitation experience ultrafast rotation during dissociation in the intermediate core-excited state. This rotation makes the vibrational progression in RIXS sensitive to the polarization of the X-ray photons. We study the water molecule, in which the effect is expressed in RIXS through the dissociative core-excited state where the vibrational scattering anisotropy is accompanied also by violation of parity selection rules for the vibrations.
\end{abstract}

\section{INTRODUCTION}

Modern molecular spectroscopy relies on two key ideas, the Born-Oppenheimer (BO) approximation and the Franck-Condon (FC) principle. The BO approximation allows for the electronic and nuclear degrees of freedom to be decoupled. The FC principle stems from a similar argument and is based on the assumption that heavy nuclei has no time to leave the equilibrium during fast electron transitions. More strictly, the FC principle implies that the dependence of the transition dipole moment on the nuclear degrees of freedom is weak in the vicinity of the vertical transition. For this reason, the dependence can usually be neglected. However, it can be very important when the molecule is core-excited to a dissociative state and the nuclear wave packet propagates far away from equilibrium. This is the case, for example, in the Resonant Inelastic X-ray Scattering (RIXS) of water molecules core-excited to the $\left|1 a_{1}^{-1} 4 a_{1}^{1}\right\rangle$ dissociative state $[1,2]$. One could expect that this geometry dependence of the dipole moment would only matter for the intensity of the so called atomic-like peak [2-6] formed in the region of dissociation far away from equilibrium. However, for the showcase water molecule presented here this dependence is prominent also for RIXS transitions which populate the low-lying vibrational states, corresponding to small deviation from equilibrium. We show that the transition dipole moment experiences ultrafast rotation in the course of the dissociation in the coreexcited state. This rotation results in a different polarization dependence at different vibrational resonances. One should mention that vibrational scattering anisotropy has been observed earlier in RIXS of oxygen molecule [7], in which it is caused by interference between resonant and

\footnotetext{
*vvdc@kth.se

† faris@kth.se
}

nonresonant scattering channels. The interference of direct ionization and resonant Auger scattering channels also makes the vibrational progression in resonant Auger spectra sensitive to the scattering angle [8-11].

We consider here the RIXS channel of $\mathrm{H}_{2} \mathrm{O}$ which induce scattering through the dissociative $\left|1 a_{1}^{-1} 4 a_{1}^{1}\right\rangle$ state into the electronic ground state. It has been previously shown $[2,12,13]$ that this RIXS channel is weakly affected by the bending mode and it is well described by considering only the coupled dynamics of the OH stretching modes. We use here the same approximation and label the ground-state vibrational levels of water $\left|n_{s}, n_{a}\right\rangle$ by quantum numbers of symmetric $\left(n_{s}\right)$ and antisymmetric $\left(n_{a}\right)$ stretching modes. It is relevant to note that the close lying vibrational states are clustered in groups, characterised by the group number $n=n_{s}+n_{a}$ [14]. Current RIXS spectroscopy, being unable to resolve the fine structure inside of each group, displays only compound peaks which we denote by $n$.

In our previous studies of the RIXS of $\mathrm{H}_{2} \mathrm{O}$, we have neglected the dependence of the transition dipole moment of core-excitation $\mathbf{d}_{c 0}(\mathbf{R})$ on the nuclear coordinates R. However, Fig. 1 shows that this dependence is strong in the case of core-excitation to a dissociative state $\left|1 a_{1}^{-1} 4 a_{1}^{1}\right\rangle$. This figure displays the $y$ and $z$ components of $\mathbf{d}_{c 0}(\mathbf{R})$ where the $y$-axis lies along the antisymmetric coordinate and the $z$-axis is oriented along the molecular symmetry axis. Most prominent dependence is seen for $d_{c 0}^{(y)}(\mathbf{R})$ which contrary to $d_{c 0}^{(z)}(\mathbf{R})$ is an antisymmetric function of $y$. As one can see from Fig. 2, this antisymmetry means opposite rotation of the transition dipole moment in the course of coherent dissociation along two pathways $[2,12,13]: H^{(1)} O----H^{(2)}$ and $H^{(2)} \mathrm{O}----H^{(1)}$. Here it is important to notice that the $\mathrm{C}_{2 v}$ symmetry of the system is preserved in quantum mechanics contrary classical mechanics where the dissociation selects only one path and breaks the symmetry. Because the symmetry is not broken in the 
course of dissociation, the RIXS process obeys the selection rules [15]. The immediate consequence of the antisymmetric dependence of $d_{c 0}^{(y)}(\mathbf{R})$ is the opening of the symmetry forbidden RIXS channel to antisymmetric vibrational states with odd quantum numbers $|00\rangle \rightarrow$ $\left|n_{s} n_{a}\right\rangle, \quad n_{a}=2 m+1$. The $\mathbf{R}$ dependence of the transition dipole moment is also associated with another physical effect. Namely, one can expect different polarization dependence of the RIXS resonances related to even and odd antisymmetric stretching states because they are populated by the orthogonal z- and y-components of $\mathbf{d}_{0 c}(\mathbf{R})$, respectively.

\section{ROTATION OF TRANSITION DIPOLE MOMENT IN THE COURSE OF DISSOCIATION}

Core-excitation to the $\left|1 s_{O}^{-1} 4 a_{1}^{1}\right\rangle$ dissociative state results in ultrafast fragmentation of the water molecule. Due to the molecular symmetry, the nuclear wave packet propagates coherently along two indistinguishable pathways $[2]$

$$
\omega+H O H \rightarrow\left\{\begin{array}{l}
H O+H \\
H+O H
\end{array}\right.
$$

following the two valleys of the core-excited PES (see Fig. 2). Let us first consider the dissociation along one pathway, where the symmetry is broken. At the ground state equilibrium geometry only the $z$-component of the transition dipole moment is non-zero in the molecular frame. The $y$-component starts to grow with elongation of one of the bonds. The physical reason for the rotation of the transition dipole moment $\mathbf{d}_{0 c}$ is the symmetry breaking in the course of the elongation of one bond. The breaking of another $\mathrm{OH}$ bond results in an opposite rotation of the transition dipole moments and, hence, opposite sign of $d_{c 0}^{(y)}$ (see Figs. 1 and 2).

To get insight into the microscopic mechanism of the rotation of the transition dipole moment we consider the evolution of the electronic wave functions in the course of the dissociation. We focus on the highest occupied and lowest unoccupied molecular orbitals (HOMO and LUMO) at equilibrium, $R_{e q}=1.81$ a.u.(see upper panels in Fig. 3) and compare them to the adiabatically related wave functions in the asymptotic region, $R=6$ a.u. Due to degeneracy, the associated $3 a_{1}$ and $4 a_{1}$ orbitals are equally populated in the asymptotic region and the ground state wave function, according to our simulations, is

$$
\begin{aligned}
& \Psi_{\mathrm{GS}}(R=\infty)=\frac{1}{\sqrt{2}}\left(\left\|3 a_{1}^{2} 4 a_{1}^{0}\right\|-\left\|3 a_{1}^{0} 4 a_{1}^{2}\right\|\right) \\
& =\frac{1}{\sqrt{2}}\left(3 a_{1}(1) 3 a_{1}(2)-4 a_{1}(1) 4 a_{1}(2)\right) \chi_{S}(1,2),
\end{aligned}
$$

Here $\|\cdots\|$ is the Slater determinant, $\alpha$ and $\beta$ are the spin functions and $\left.\chi_{S}=[\alpha(1) \beta(2)-\beta(1) \alpha(2)] / \sqrt{(} 2\right)$ is the singlet spin function. To avoid unnecessarily cumbersome notations, we keep the orbital assignment $3 a_{1}$ and $4 a_{1}$ of the $\mathrm{C}_{2 v}$ point group in spite that the symmetry is broken along the selected dissociation channel. Calculations show that the orbitals in the asymptotic limit have equal contributions from the atomic orbitals $2 p_{z^{\prime}}^{(O)}$ and $1 s^{(H)}$ (Fig. 3)

$$
\left.\begin{array}{l}
4 a_{1} \\
3 a_{1}^{\prime}
\end{array}\right\}=\frac{1}{\sqrt{2}}\left[2 p_{z^{\prime}}^{(O)} \pm 1 s^{(H)}\right]
$$

here the $z^{\prime}$-axis is perpendicular to the intact $\mathrm{OH}$ bond (see Fig. 3). Substitution of this equation into (2) gives the following asymptote for $\Psi_{\mathrm{GS}}$, expressed in singly occupied molecular orbitals (SOMOs)

$$
\begin{aligned}
\Psi_{\mathrm{GS}}(R=\infty) & =\frac{1}{\sqrt{2}}\left(\left\|1 s^{(O)} \alpha 1 s^{(O)} \beta \cdots 2 p_{z^{\prime}}^{(O)} \alpha 1 s^{(H)} \beta\right\|\right. \\
& \left.-\left\|1 s^{(O)} \alpha 1 s^{(O)} \beta \cdots 2 p_{z^{\prime}}^{(O)} \beta 1 s^{(H)} \alpha\right\|\right) .
\end{aligned}
$$

Let us turn to the wave function of the core-excited state $\Psi_{\mathrm{CE}}$. At equilibrium geometry, the core-excitation populates the former LUMO $4 a_{1}$, and the HOMO $3 a_{1}$ remains doubly occupied. In the asymptotic region $(R=$ $\infty$.) in contrast to the ground state, the HOMO and the SOMO in the core-excited state are fully localized on the oxygen or dissociated hydrogen atoms, respectively (Fig. 3)

$$
3 a_{1}=2 p_{z^{\prime}}^{(O)}, \quad 4 a_{1}=1 s^{\left(H_{1}\right)} .
$$

Thus the asymptote of the core-excited singlet wavefunction reads

$$
\begin{aligned}
\Psi_{\mathrm{CE}}(R=\infty) & =\frac{1}{\sqrt{2}}\left(\left\|1 s^{(O)} \alpha \cdots 2 p_{z^{\prime}}^{(O)} \alpha 2 p_{z^{\prime}}^{(O)} \beta 1 s^{\left(H_{1}\right)} \beta\right\|\right. \\
& \left.-\left\|1 s^{(O)} \beta \cdots 2 p_{z^{\prime}}^{(O)} \alpha 2 p_{z^{\prime}}^{(O)} \beta 1 s^{\left(H_{1}\right)} \alpha\right\|\right) .
\end{aligned}
$$

Therefore the transition dipole moment $\mathbf{d}_{c 0}=$ $\left\langle\Psi_{\mathrm{CE}}|\mathbf{d}| \Psi_{\mathrm{GS}}\right\rangle \approx\left\langle\left|1 s^{(O)}\right| \mathbf{r} \mid 2 p_{z^{\prime}}^{(O)}\right\rangle$ at $R=\infty$ has only a $z^{\prime}$ component. Thus $\mathbf{d}_{c 0}$ rotates in the course of dissociation from the $z$ direction at $R=R_{e q}$ to the $z^{\prime}$ direction at $R=\infty$ as shown schematically in Fig. 2. The opposite rotation of $\mathbf{d}_{c 0}$ in the left and right half planes with respect to the $z$ axis (Fig. 2) explains the antisymmetry of $d_{c 0}^{(y)}$ (Fig. 1).

\section{A. Theory of RIXS including rotation of the transition dipole moment}

Here we outline the theory of RIXS which takes into account the rotation of the transition dipole moments for randomly oriented molecules. To carry out the averaging over molecular orientations let us remind ourselves that the scattering process to the final state $f$ is defined by the scalar products of the polarization vectors $\mathbf{e}$ and $\mathbf{e}^{\prime}$ with 
the transition dipole moments of core-excitation $\mathbf{d}_{c 0}=$ $\mathbf{d}_{c 0}(\mathbf{R})$ and decay $\mathbf{d}_{f c}=\mathbf{d}_{f c}(\mathbf{R})$, respectively

$$
\left(\mathbf{e} \cdot \mathbf{d}_{c 0}\right)=\sum_{i} e_{i} d_{c 0}^{(i)}, \quad\left(\mathbf{e}^{\prime} \cdot \mathbf{d}_{f c}\right)=\sum_{k} e_{k}^{\prime} d_{f c}^{(k)} .
$$

Instead of averaging over molecular orientations, we fix the molecular frame and perform an averaging over the orientations of polarization vectors. Eq. (7) allows to get the following expression for the RIXS cross-section [15, $16]$

$$
\begin{aligned}
& \sigma\left(\omega^{\prime}, \omega, \theta\right)=\sum_{i j k l} \overline{e_{i} e_{j} e_{k}^{\prime} e_{l}^{\prime}} \sigma_{j l k i}, \\
& \sigma_{j l k i}=\sum_{\nu_{f}}\left(F_{\nu_{f} 0}^{l j}\right)^{\dagger} F_{\nu_{f} 0}^{k i} \Delta\left(\varepsilon, \Gamma_{f}\right) \\
& =\sum_{\nu_{f}}\left\langle\Psi_{l j}(0) \mid \nu_{f}\right\rangle\left\langle\nu_{f} \mid \Psi_{k i}(0)\right\rangle \Delta\left(\varepsilon, \Gamma_{f}\right), \\
& F_{\nu_{f}}^{k i}=-\imath\left\langle\nu_{f} \mid \Psi_{k i}(0)\right\rangle .
\end{aligned}
$$

Here $\varepsilon=\omega-\omega^{\prime}-\omega_{f 0}-\epsilon_{\nu_{f}}+\epsilon_{\nu_{0}}, \theta=\angle\left(\mathbf{e}^{\prime}, \mathbf{e}\right), \Delta(\Omega, \Gamma)=$ $\Gamma /\left[\pi\left(\Omega^{2}+\Gamma^{2}\right)\right]$. The partial scattering amplitude $F_{\nu_{f}}^{k i}$ is the projection of the wave packet $\left|\Psi_{k i}(0)\right\rangle$

$$
\begin{aligned}
&\left|\Psi_{k i}(0)\right\rangle=\imath \sum_{\nu_{c}} \frac{d_{f c}^{(k)}\left|\nu_{c}\right\rangle\left\langle\nu_{c}\left|d_{c 0}^{(i)}\right| \nu_{0}\right\rangle}{\omega-\omega_{c 0}+\epsilon_{\nu_{0}}-\epsilon_{\nu_{c}}+\imath \Gamma} \\
&=d_{f c}^{(k)} \int_{0}^{\infty} e^{\imath\left(\omega-\omega_{c 0}+\epsilon_{\nu_{0}}+\imath \Gamma\right) t}\left|\psi_{c}^{(i)}(t)\right\rangle d t \\
&\left|\psi_{c}^{(i)}(t)\right\rangle=e^{-\imath h_{c} t} d_{c 0}^{(i)}|0\rangle
\end{aligned}
$$

onto the final vibrational state $\left|\nu_{f}\right\rangle$. In simulations, we use a time-dependent representation for $\sigma\left(\omega^{\prime}, \omega, \theta\right)$ which can be obtained from Eqs. (8) and (9) using $\Delta(\Omega, \Gamma)=$ $\operatorname{Re} \int_{0}^{\infty} \exp (\imath(\Omega+\imath \Gamma)) d t / \pi$. Averaging over polarization vectors $[15,16]$ with fixed angle $\theta=\angle\left(\mathbf{e}^{\prime}, \mathbf{e}\right)$

$$
\begin{aligned}
\overline{e_{i} e_{j} e_{k}^{\prime} e_{l}^{\prime}} & =\frac{1}{6}\left[\delta_{i j} \delta_{k l}\left(1-\cos ^{2} \theta\right)\right. \\
& \left.+\frac{1}{5}\left(\delta_{i j} \delta_{k l}+\delta_{i k} \delta_{j l}+\delta_{i l} \delta_{j k}\right)\left(3 \cos ^{2} \theta-1\right)\right]
\end{aligned}
$$

results in the following equation for the RIXS crosssection

$$
\begin{aligned}
& \sigma\left(\omega^{\prime}, \omega, \theta\right)=\frac{1}{15}\left[\left(1+2 \cos ^{2} \theta\right)\left(\sigma_{z z z z}+\sigma_{y y y y}\right)\right. \\
& +\left(2-\cos ^{2} \theta\right)\left(\sigma_{z y y z}+\sigma_{y z z y}\right) \\
& \left.+\frac{1}{2}\left(3 \cos ^{2} \theta-1\right)\left(\left[\sigma_{y y z z}+\sigma_{z z y y}\right]+\left[\sigma_{z y z y}+\sigma_{y z y z}\right]\right)\right] .
\end{aligned}
$$

In the FC approximation when $R$-dependence is neglected, only $\sigma_{z z z z}$ contributes to the RIXS cross-section. The rotation of the transition dipole moments, in the course of nuclear dynamics in the core-excited state, is accounted for by the following tensor components $\sigma_{j l k i} \equiv$ $\sigma_{j l k i}\left(\omega^{\prime}, \omega\right)$ of the cross-section

$$
\begin{aligned}
& \sigma_{i k k i}=\frac{1}{\pi} \operatorname{Re} \int_{0}^{\infty} e^{\imath\left(\omega-\omega^{\prime}-\omega_{f 0}+\epsilon_{\nu_{0}}+\imath \Gamma_{f}\right) t} \sigma_{i k k i}(t) d t \\
& \sigma_{j l k i}+\sigma_{i k l j} \\
& =\frac{1}{\pi} \operatorname{Re} \int_{0}^{\infty} e^{\imath\left(\omega-\omega^{\prime}-\omega_{f 0}+\epsilon_{\nu_{0}}+\imath \Gamma_{f}\right) t}\left(\sigma_{j l k i}(t)+\sigma_{i k l j}(t)\right) d t, \\
& \sigma_{j l k i}(t)=\left\langle\Psi_{l j}(0) \mid \Psi_{k i}(t)\right\rangle, \quad\left|\Psi_{k i}(t)\right\rangle=e^{-\imath h_{f} t}\left|\Psi_{k i}(0)\right\rangle .
\end{aligned}
$$

Note that the indices $i$ and $j$ denote the Cartesian coordinates of the transition dipole moment of core-excitation whereas $k$ and $l$ refer to the decay transition.

Typically, the X-ray spectrometer collects the signal from the scattered photons of all polarizations $\mathbf{e}^{\prime}$. Therefore, we need to average over different orientations of $\mathbf{e}^{\prime}$ around the momentum vector of the scattered photon $\mathbf{k}^{\prime}$ with $\overline{\left(\mathbf{e} \cdot \mathbf{e}^{\prime}\right)^{2}}=\left(1-\cos ^{2} \chi\right) / 2$

$$
\begin{aligned}
& \sigma\left(\omega^{\prime}, \omega, \chi\right)=\frac{1}{30}\left[2\left(2-\cos ^{2} \chi\right)\left(\sigma_{z z z z}+\sigma_{y y y y}\right)\right. \\
& +\left(3+\cos ^{2} \chi\right)\left(\sigma_{z y y z}+\sigma_{y z z y}\right) \\
& \left.+\frac{1}{2}\left(1-3 \cos ^{2} \chi\right)\left(\left[\sigma_{y y z z}+\sigma_{z z y y}\right]+\left[\sigma_{z y z y}+\sigma_{y z y z}\right]\right)\right],
\end{aligned}
$$

where $\chi=\angle\left(\mathbf{k}^{\prime}, \mathbf{e}\right)$.

\section{COMPUTATIONAL DETAILS}

The PESs and transition dipole moment surfaces were calculated using the restricted active space self-consistent field (RASSCF) [17] method with second-order perturbation theory (RASPT2) correction in the MOLCAS version $8.2[18,19]$ computational software. The simulations were performed in $\mathrm{C}_{s}$ symmetry using the ANORCC [20] basis set. The RAS state interaction program (RASSI) [21, 22] was used to compute the transition dipole moments. The 2D PESs of the ground and the lowest core-excited $\left|1 s_{O}^{-1} 4 a_{1}^{1}\right\rangle$ states, as well as transition dipole moments were computed in the space of valence coordinates $R_{1}$ and $R_{2}$. To calculate the PES of the coreexcited state the "highly-excited-state" scheme (HEXS) was employed [23]. This way we could exclude configurations with fully occupied RAS1 space from the calculation of the core-excited state. Further details can be found in our previous studies $[2,24]$. The nuclear wave packets (9) and (12) needed for the partial cross-sections $\sigma_{j l k i}(12)$ were computed using the second order difference propagation scheme using the discrete variable representation. The time-dependent Schrödinger equation with 2D Hamiltonians [25] $(\mathrm{i}=0, \mathrm{c})$

$$
h_{i}=-\frac{1}{2 \mu}\left(\partial_{R_{1}^{2}}^{2}+\partial_{R_{2}^{2}}^{2}\right)-\frac{\cos \theta_{0}}{m_{O}} \partial_{R_{1} R_{2}}^{2}+V_{i}\left(R_{1}, R_{2}\right)
$$


was solved with the propagation step $\Delta t=5 \times 10^{-4}$ fs. The kinetic energy operator was represented in the position space using a fourth order numerical difference formula. All calculations were performed with a modified version of the e $S P$ ec code used in references $[1,2,12,13]$. To avoid unphysical reflections at the edges of the numerical grid, absorbing boundary conditions were included in all propagations.

\section{A. Violation of parity selection rules}

In order to show the breakdown of the selection rules caused by $\mathbf{R}$ dependence of the transition dipole moment, we notice that the intensity of the RIXS transition $|0,0\rangle \rightarrow\left|\nu_{f}\right\rangle \equiv\left|n_{s}, n_{a}\right\rangle$ (Eq. (8)) is defined by the product

$$
\left\langle\Psi_{j l}(0) \mid n_{s}, n_{a}\right\rangle\left\langle n_{s}, n_{a} \mid \Psi_{k i}(0)\right\rangle .
$$

Hence, the parity of the wave packet $\left|\Psi_{k i}(0)\right\rangle$ is given by the parity of the product of the components of transition dipole moments $d_{f c}^{(k)} \times d_{c 0}^{(i)}$. As one can see from Fig. 1 the $y$ component of the transition dipole moments is an antisymmetric function of $y$ while the $z$ component is symmetrical with respect to $y$. Thus the symmetryforbidden decay channel $|0,0\rangle \rightarrow\left|n_{s}, 2 m+1\right\rangle$ to odd antisymmetric stretching mode levels is opened solely by antisymmetric $\left|\Psi_{z y}(0)\right\rangle$ and $\left|\Psi_{y z}(0)\right\rangle$ wave packets (see Fig. 4). This allows us to write the cross-section as a sum of two contributions

$$
\sigma\left(\omega^{\prime}, \omega, \chi\right)=\sigma_{\text {even }}\left(\omega^{\prime}, \omega, \chi\right)+\sigma_{\text {odd }}\left(\omega^{\prime}, \omega, \chi\right) .
$$

The first term in the above equation corresponds to the transitions to even antisymmetric final vibrational levels $\left(\nu_{f}=\left(n_{s}, 2 m\right)\right)$ while the second term corresponds to the transitions to odd final states $\left(\nu_{f}=\left(n_{s}, 2 m+1\right)\right)$, which are forbidden when $\mathbf{d}_{c 0}(\mathbf{R}) \equiv$ const,

$$
\begin{aligned}
\sigma_{\text {even }}\left(\omega^{\prime}, \omega, \chi\right) & =\frac{1}{30}\left[2\left(2-\cos ^{2} \chi\right)\left(\sigma_{z z z z}+\sigma_{y y y y}\right)\right. \\
& \left.+\frac{1}{2}\left(1-3 \cos ^{2} \chi\right)\left[\sigma_{y y z z}+\sigma_{z z y y}\right]\right], \\
\sigma_{\text {odd }}\left(\omega^{\prime}, \omega, \chi\right) & =\frac{1}{30}\left[\left(3+\cos ^{2} \chi\right)\left(\sigma_{z y y z}+\sigma_{y z z y}\right)\right. \\
& \left.+\frac{1}{2}\left(1-3 \cos ^{2} \chi\right)\left[\sigma_{z y z y}+\sigma_{y z y z}\right]\right] .
\end{aligned}
$$

One should notice that according to Eq. (9) the origin of the antisymmetry of $\left|\Psi_{z y}(0)\right\rangle$ is the antisymmetric $y$ dependence of the $y$ component of the transition dipole moment $d_{c 0}^{(y)}$, in contrast to $\left|\Psi_{y z}(0)\right\rangle$ which gets this asymmetry from the decay transition dipole moment $d_{f c}^{(y)}$.

The total $\sigma$ and partial $\left(\sigma_{\text {even }}, \sigma_{\text {odd }}\right)$ cross-sections are shown for two different detection angles in Fig. 5. We can see that $\sigma_{\text {odd }}$ does not contribute to elastic peak $(n=0)$. Notice that our simulations do not take into account the Thomson scattering term [16]. Due to this, the elastic line is not properly described for $\chi=90^{\circ}$, but it is accurate for $\chi=0^{\circ}$, where the Thomson term is absent.

Each insert in Fig. 5 displays the comparison between the computed total RIXS cross-sections and one computed within the FC approximation (which is not sensitive to polarization). One can see that when we go beyond the FC approximation there is an enhancement of the higher vibrational peaks in the progression with respect to peak $n=1\left(\omega-\omega^{\prime} \approx 0.45 \mathrm{eV}\right)$. Also, the intensity of the atomic-like peak, which arises from decay of the $\mathrm{OH}$ fragment [2], is increased. One should notice that the atomic-like peak overlaps with the pseudoatomic peak which arises due to the electronic inelastic scattering channel to the first valence-excited state [24] $\left|1 b_{1}^{-1} 4 a_{1}^{1}\right\rangle$, which has the same dissociation limit as the ground state.

A look at the full profile for the inelastic losses reveals that we have not only a violation of the selection rules (i.e. $\sigma_{\text {odd }} \neq 0$ ) but we clearly see that there is a strong dependence of the relative intensity of $\sigma_{\text {odd }}$ and $\sigma_{\text {even }}$ on the polarization of the photons. This effect could be the subject of future high-resolution RIXS measurements and will be discussed in more detail in the next section.

However, the vibrational scattering anisotropy can also be addressed using the presently available RIXS resolution, which only allows us to resolve vibrational peaks $\sigma_{n}\left(\omega^{\prime}, \omega, \chi\right)$ belonging to different groups characterized by group number $n=n_{s}+n_{a}$. In this case, it would be possible to observe this effect considering the angular dependence of the ratio of the area of the $n$-th peak to the area of the first peak $n=1$

$$
\frac{S_{n}(\chi)}{S_{1}(\chi)}, \quad S_{n}=\int_{\omega^{\prime} \in n} d \omega^{\prime} \sigma\left(\omega^{\prime}, \omega, \chi\right) .
$$

For the water molecule, considered here, this dependence is rather weak (Fig. 6). Indeed, each unresolved $n$-th RIXS peak contains contributions from both even and odd levels, which intensities have opposite polarization dependence suppressing strongly the angular dependence (see Fig. 6) seen for symmetric and antisymmetric resonances inside of the $n$-th group (Fig. 7).

\section{B. Polarization dependence of symmetric and antisymmetric resonances}

Eq. (17) shows that even and odd antisymmetric vibrational states have qualitatively different polarization dependence, as discussed in the previous section. This effect becomes evident when we consider the ratio

$$
\rho^{(1)}(\chi)=\frac{\sigma_{\text {even }}^{(1,0)}\left(\omega^{\prime}, \omega, \chi\right)}{\sigma_{\text {odd }}^{(0,1)}\left(\omega^{\prime}, \omega, \chi\right)}
$$

of the peak intensities for "symmetry allowed" $|0,0\rangle \rightarrow$ $|1,0\rangle$ and "symmetry forbidden" $|0,0\rangle \rightarrow|0,1\rangle$ RIXS 
transitions (see Fig. 7). In this equation, the superscripts $(1,0)$ and $(0,1)$ designate the final vibrational state $\left(n_{s}, n_{a}\right)$. Such an anomalously strong polarization dependence is in principle observable, but super-high resolution RIXS measurements are needed to resolve the close-lying $(1,0)$ and $(0,1)$ states with energy spacing of about $9 \mathrm{meV}$. Considering recent experimental development, this energy resolution could be achieved within a few years [26-29].

\section{CONCLUSIONS}

We found a strong dependence of the transition dipole moment on geometric distortion in the core-excited water molecule. The transition dipole moment experiences a rotation in the molecular frame in the course of ultrafast dissociation in the core-excited state. This rotation opens the symmetry forbidden RIXS channel to odd vibrational levels of the antisymmetric stretching mode, since the $y$-component of the transition dipole moment is an antisymmetric function of the coordinate. This results in strong non-FC effects in the RIXS profile, in particular in a pronounced polarization dependence of the vibrational progression which is absent within the FC approximation. The effect can be observed experimentally by measuring the ratio of symmetry allowed and symmetry forbidden vibrational peaks, if the spectral resolution is high enough $(\sim 10 \mathrm{meV})$. When the resolution is not high enough, the polarization dependence can be detected measuring the ratio of the area of the $n$ vibrational resonance to the area of the first vibrational peak. We expect that the effect, studied here, can also be present for bound core-excited states and in other molecules.

\section{ACKNOWLEDGMENTS}

This work was supported by the Swedish Research Council (VR), the Knut and Alice Wallenberg foundation (Grant No. KAW-2013.0020), Carl Tryggers Stiftelse, and Russian Scientific Foundation (project 16-12-10109). V. V. C. and R. C. C. acknowledge the Conselho Nacional de Desenvolvimento Cientfico e Tecnolgico (CNPq - Brazil). Quantum chemical calculations and numerical simulations were performed on the resources provided by the Swedish National Infrastructure for Computing (SNIC).
[1] R. C. Couto, V. Vaz da Cruz, E. Ertan, S. Eckert, M. Fondell, M. Dantz, B. O'Cinneide, T. Schmitt, A. Pietzsch, F. F. Guimarães, H. Ågren, F. Gel'mukhanov, M. Odelius, V. Kimberg, and A. Föhlisch, Nat. Commun. 8, 14165 (2017).

[2] V. Vaz da Cruz, E. Ertan, R. C. Couto, S. Eckert, M. Fondell, M. Dantz, B. Kennedy, T. Schmitt, A. Pietzsch, F. F. Guimaraes, H. Agren, F. Gel'mukhanov, M. Odelius, A. Fohlisch, and V. Kimberg, Phys. Chem. Chem. Phys. 19, 19573 (2017).

[3] F. Gel'mukhanov and H. Ågren, Phys. Rev. A 54, 379 (1996).

[4] P. Sałek, V. Carravetta, F. Gel'mukhanov, and H. Ågren, J. Chem. Phys. 116, 629 (2002).

[5] P. Sałek, F. Gel'mukhanov, and H. Ågren, Phys. Rev. A 59, 1147 (1999).

[6] A. Pietzsch, Y.-P. Sun, F. Hennies, Z. Rinkevicius, H. O. Karlsson, T. Schmitt, V. N. Strocov, J. Andersson, B. Kennedy, J. Schlappa, A. Föhlisch, J.-E. Rubensson, and F. Gel'mukhanov, Phys. Rev. Lett. 106, 153004 (2011).

[7] Y.-P. Sun, Q. Miao, A. Pietzsch, F. Hennies, T. Schmitt, V. N. Strocov, J. Andersson, B. Kennedy, J. Schlappa, A. Föhlisch, F. Gel'mukhanov, and J.-E. Rubensson, Phys. Rev. Lett. 110, 223001 (2013).

[8] C. Miron, V. Kimberg, P. Morin, C. Nicolas, N. Kosugi, S. Gavrilyuk, and F. Gel'mukhanov, Phys. Rev. Lett. 105, 093002 (2010).

[9] E. Kukk, J. D. Bozek, W.-T. Cheng, R. F. Fink, A. A. Wills, and N. Berrah, J. Chem. Phys. 111, 9642 (1999).

[10] I. Hjelte, L. Karlsson, S. Svensson, A. D. Fanis, V. Carravetta, N. Saito, M. Kitajima, H. Tanaka, H. Yoshida, A. Hiraya, I. Koyano, K. Ueda, and M. N. Piancastelli,
J. Chem. Phys. 122, 084306 (2005).

[11] A. Lindblad, V. Kimberg, J. Sderstrm, C. Nicolas, O. Travnikova, N. Kosugi, F. Gel'mukhanov, and C. Miron, New Journal of Physics 14, 113018 (2012).

[12] N. Ignatova, V. Vaz da Cruz, R. C. Couto, E. Ertan, A. Zimin, F. F. Guimarães, S. Polyutov, H. Ågren, V. Kimberg, M. Odelius, and F. Gel'mukhanov, Sci. Rep. 7, 43891 (2017).

[13] N. Ignatova, V. V. da Cruz, R. C. Couto, E. Ertan, M. Odelius, H. Ågren, F. F. Guimarães, A. Zimin, S. P. Polyutov, F. Gel'mukhanov, and V. Kimberg, Phys. Rev. A 95, 042502 (2017).

[14] A. G. Császár, E. Mátyus, T. Szidarovszky, L. Lodi, N. F. Zobov, S. V. Shirin, O. L. Polyansky, and J. Tennyson, J. Quant. Spectrosc. Radiat. Transfer 111, 1043 (2010).

[15] F. Gel'mukhanov and H. Ågren, Phys. Rev. A 49, 4378 (1994).

[16] F. Gel'mukhanov and H. Ågren, Phys. Rep. 312, 87 (1999).

[17] P.-Å. Malmqvist, A. Rendell, and B. O. Roos, J. Phys. Chem. 94, 5477 (1990).

[18] F. Aquilante, L. De Vico, N. Ferré, G. Ghigo, P.-Å. Malmqvist, P. Neogrády, T. B. Pedersen, M. Pitonak, M. Reiher, B. O. Roos, L. Serrano-Andrés, M. Urban, V. Veryazov, and R. Lindh, J. Comp. Chem. 31, 224 (2010).

[19] F. Aquilante, J. Autschbach, R. K. Carlson, L. F. Chibotaru, M. G. D elcey, L. De Vico, I. Fdez. Galván, N. Ferré, L. M. Frutos, L. Gagliardi, M. Garavelli, A. Giussani, C. E. Hoyer, G. Li Manni, H. Lischka, D. Ma, P.-Å. Malmqvist, T. Müller, A. Nenov, M. Olivucci, T. B. Pedersen, D. Peng, F. Plasser, B. Pritchard, M. Reiher, I. Rivalta, I. Schapiro, J. Segarra-Martí, M. Sten- 
rup, D. G. Truhlar, L. Ungur, A. Valentini, S. Vancoillie, V. Veryazov, V. P. Vysotskiy, O. Weingart, F. Zapata, and R. Lindh, J. Comp. Chem. 37, 506 (2016).

[20] B. O. Roos, R. Lindh, P.-Å. Malmqvist, V. Veryazov, and P.-O. Widmark, J. Phys. Chem. A 108, 2851 (2004).

[21] P.-Å. Malmqvist and B. O. Roos, Chem. Phys. Lett. 155, 189 (1989).

[22] P.-Å. Malmqvist, B. O. Roos, and B. Schimmelpfennig, Chem. Phys. Lett. 357, 230 (2002).

[23] M. Guo, L. K. Sorensen, M. G. Delcey, R. V. Pinjari, and M. Lundberg, Phys. Chem. Chem. Phys. 18, 3250 (2016).

[24] E. Ertan, V. Savchenko, N. Ignatova, V. Vaz da Cruz, R. C. Couto, S. Eckert, M. Fondell, M. Dantz, B. Kennedy, T. Schmitt, A. Pietzsch, A. Föhlisch, F. Gel'mukhanov, M. Odelius, and V. Kimberg, Phys.
Chem. Chem. Phys. 20, 14384 (2018).

[25] P. Jensen, J. Chem. Soc., Faraday Trans. 2 84, 1315 (1988).

[26] T. Schmitt, F. M. F. de Groot, and J.-E. Rubensson, J. Synchrotron Radiat. 21, 1065 (2014).

[27] J. Dvorak, I. Jarrige, V. Bisogni, S. Coburn, and W. Leonhardt, Rev. Sci. Instrum. 87, 115109 (2016).

[28] Information on the upcoming VERITAS beamline for high-resolution RIXS., "https://www.maxiv.lu. se/accelerators-beamlines/beamlines/veritas," Accessed: 2017-02-28.

[29] A. Pietzsch, A. Sokolov, T. Blume, S. Neppl, F. Senf, F. Siewert, and A. Föhlisch, Synchrotron Radiat. News 31, 20 (2018). 
a)

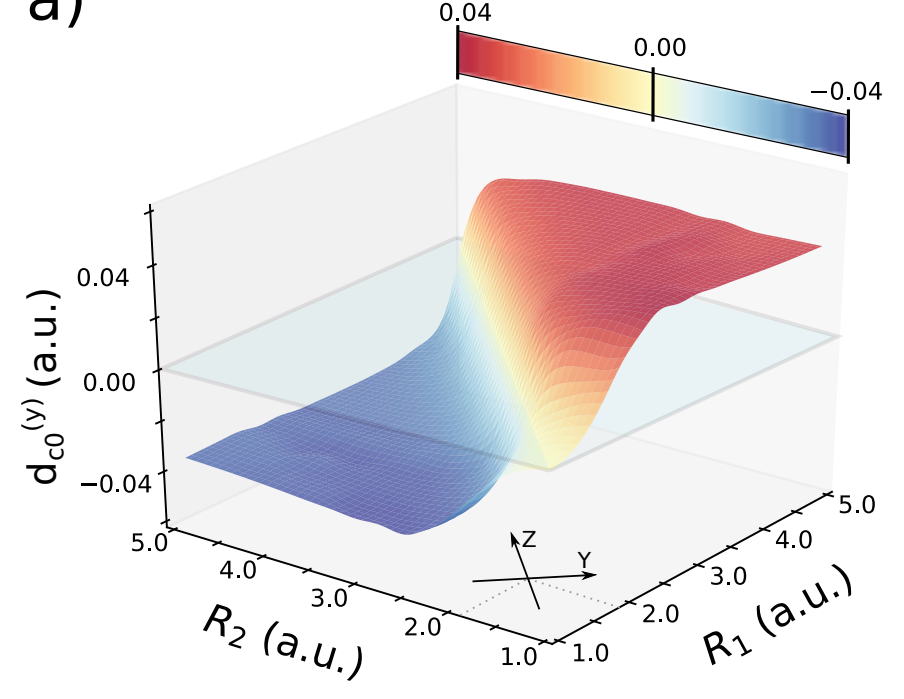

b)

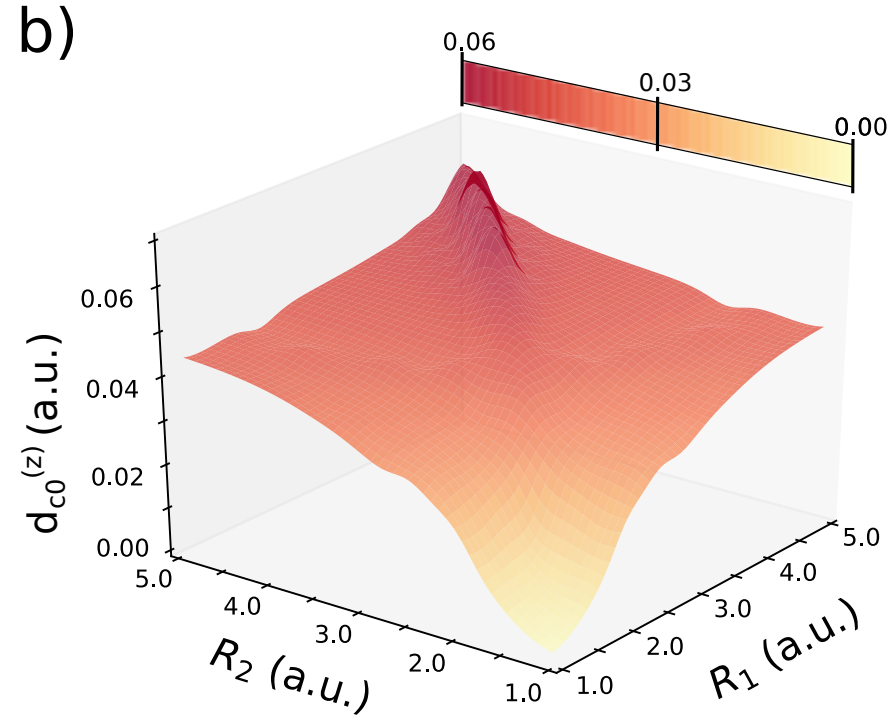

FIG. 1. Surfaces of the components of the transition dipole moment $\mathbf{d}_{c 0}$ as functions of the bond lengths $R_{1}$ and $R_{2}$. Panel a) displays the anti-symmetric $y$ component while panel b) shows the symmetric $z$ component.

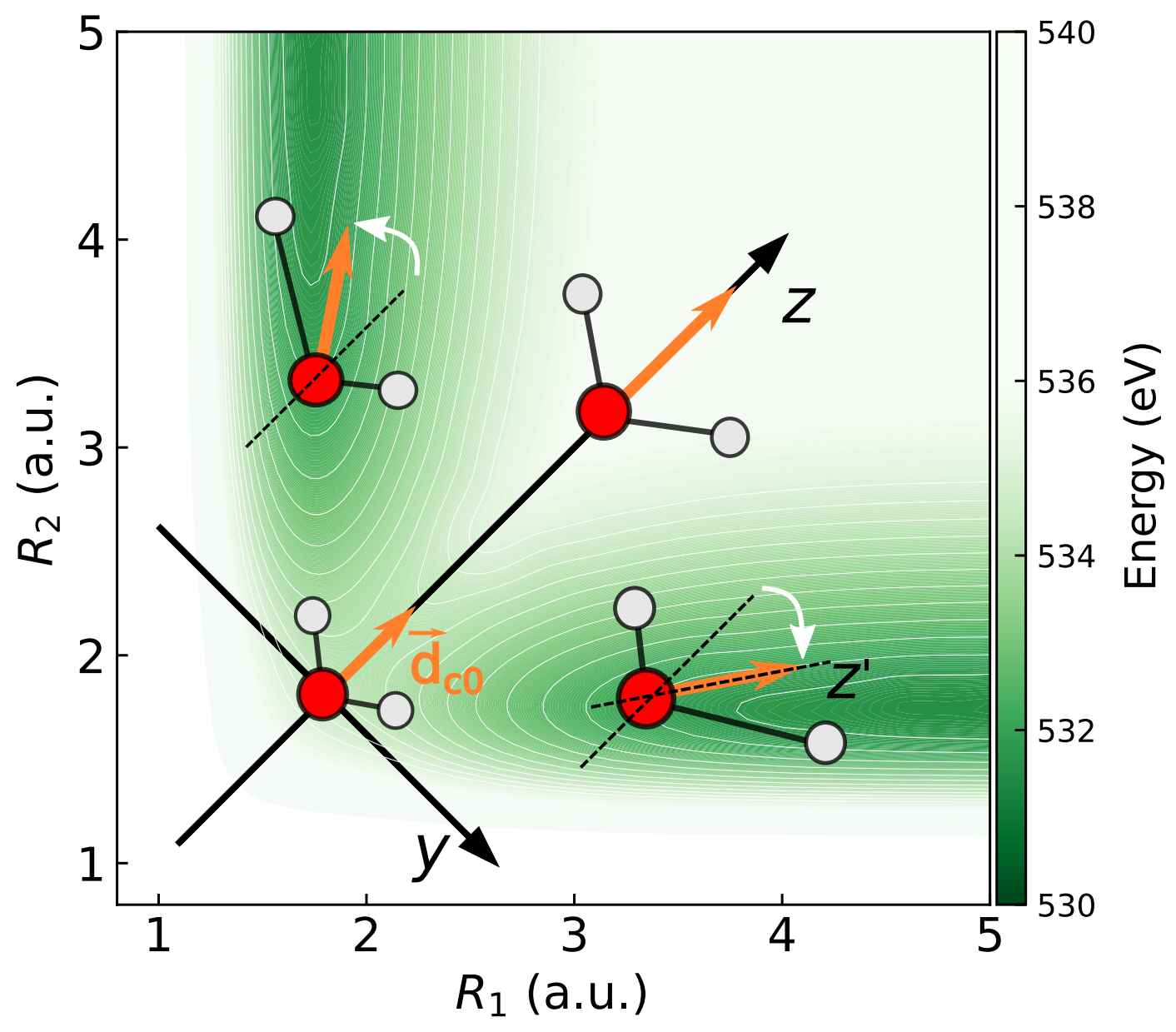

FIG. 2. Schematic picture of opposite rotation of the transition dipole moment in the course of coherent dissociation along different pathways. The green colormap displays the potential energy surface of the dissociative $\left|O 1 s^{-1} 4 a_{1}^{1}\right\rangle$ core-excited state as function of the bond lengths $R_{1}$ and $R_{2}$. The transition dipole moment $\mathbf{d}_{c 0}$ is shown by the orange arrows. 


\section{Ground State}

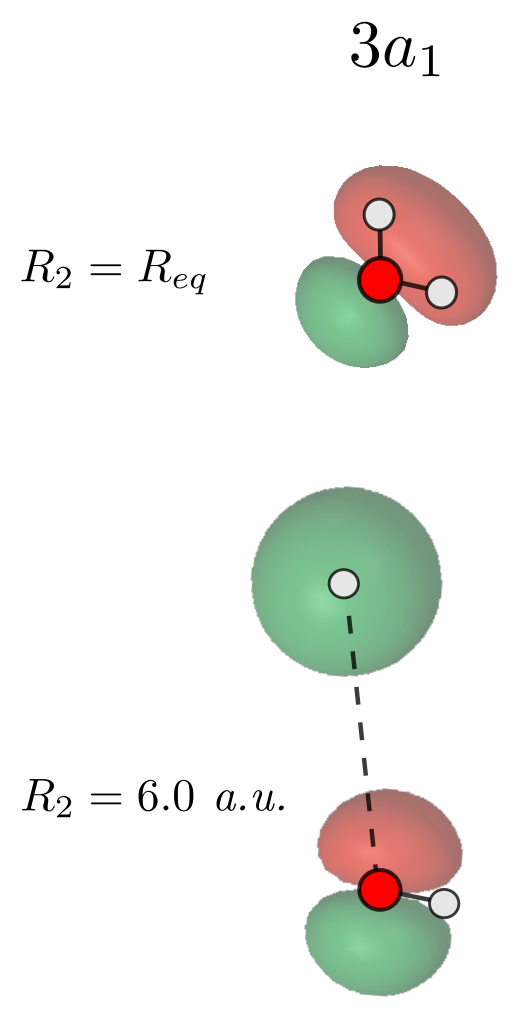

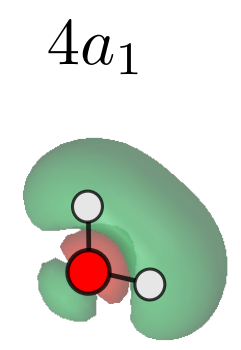

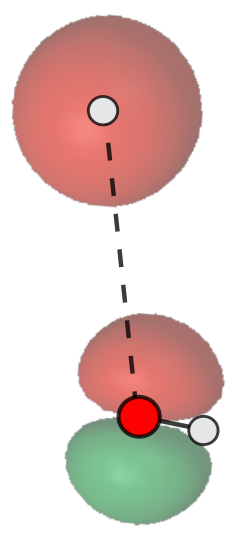

$\left|\mathrm{O} 1 \mathrm{~s}^{-1} 4 a_{1}^{1}\right\rangle$
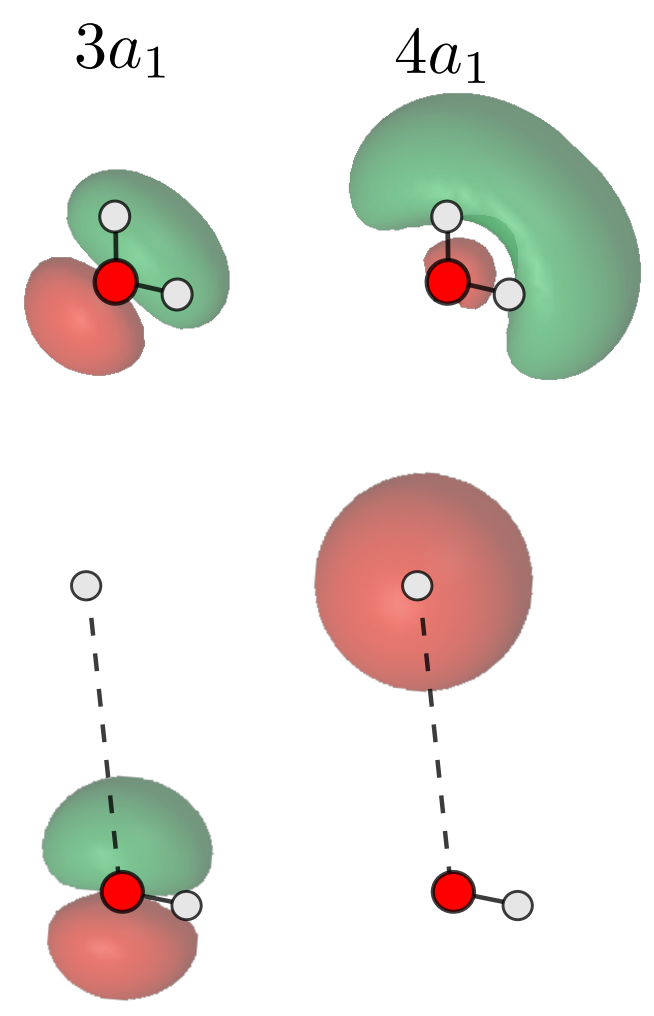

FIG. 3. Natural orbitals involved in the dissociation of the $\mathrm{OH}$ bond. The orbitals are extracted from the multi-electronic RASSCF wavefunctions of the ground state and the $\left|O 1 s^{-1} 4 a_{1}\right\rangle$ core-excited state. The upper row displays the $3 a_{1}$ and $4 a_{1}$ orbitals at equilibrium geometry $R_{1}=R_{2}=R_{e q}=1.81$ a.u. while the bottom row shows the same in the region of dissociation $R_{1}=R_{e q}=1.81$ a.u., $R_{2}=6$ a.u. 


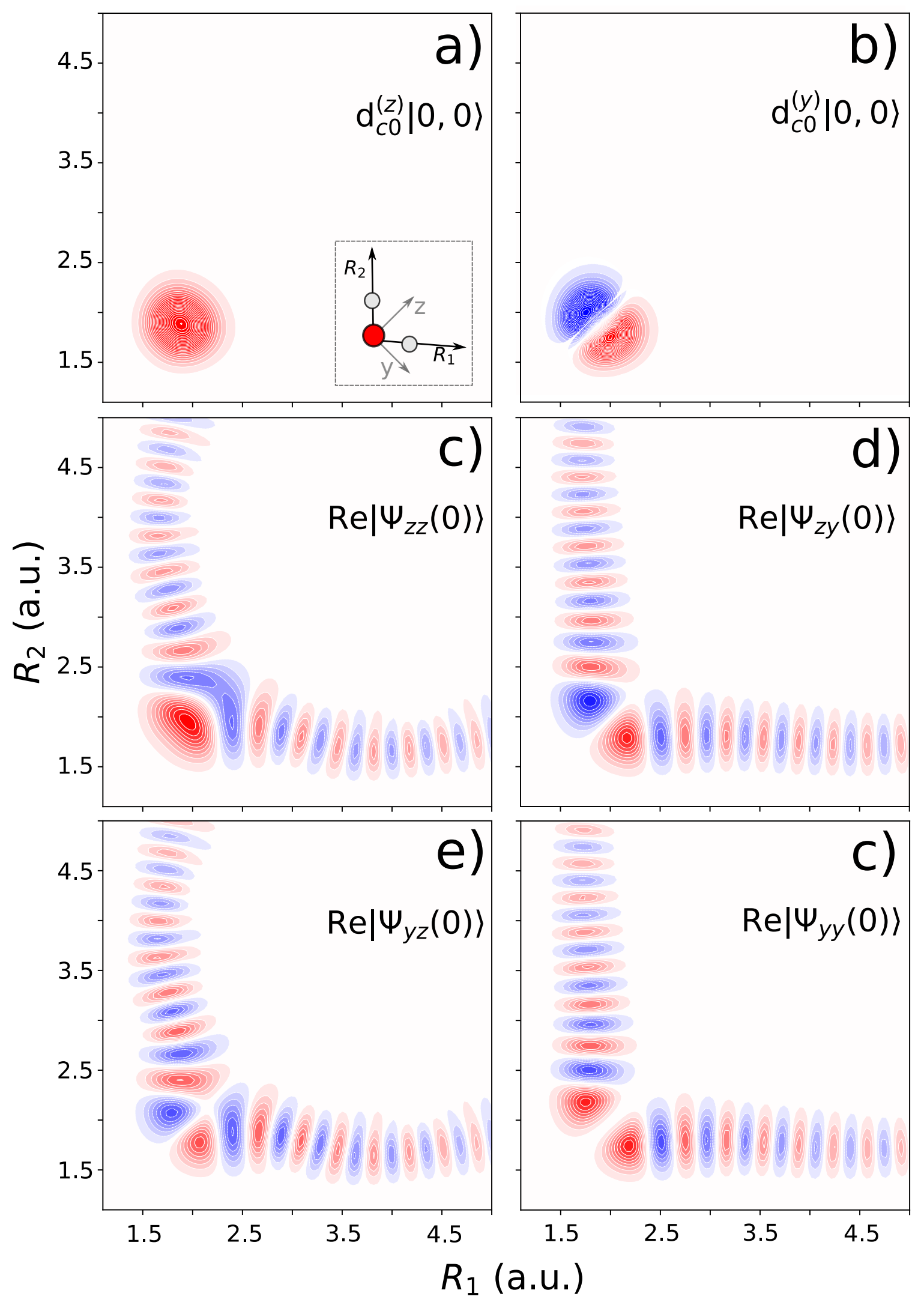

FIG. 4. Panels a) and b) show how the $z$ - and $y$-components of the transition dipole moment effectively change the symmetry of the initial vibrational state. Panels c)-f) show that contrary to $\Psi_{z z}(0)$ and $\Psi_{y y}(0)$ the y-component of $\mathbf{d}_{c 0}$ makes antisymmetric the wave packets $\Psi_{z y}(0)$ and $\Psi_{y z}(0)$. 

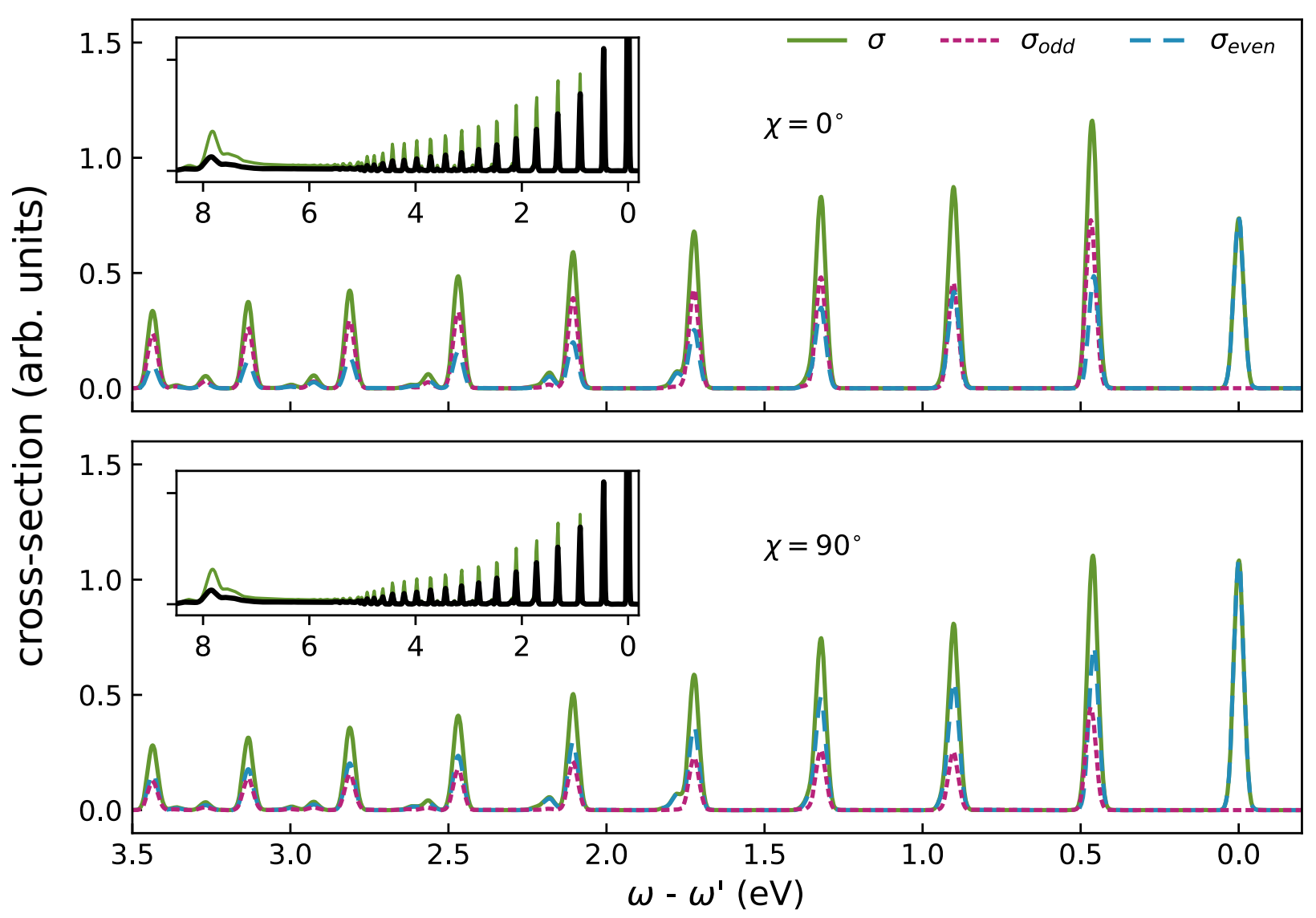

FIG. 5. Total cross-section $\sigma$ (solid green) and partial cross-sections $\sigma_{\text {odd }}$ (dotted red) and $\sigma_{\text {even }}$ (dashed blue) (Eq. 17) for $\chi=0^{\circ}$ (upper panel) and $\chi=90^{\circ}$ (lower panel). The inserts in both panels compare the strict $\sigma$ (thin green) and $\sigma$ computed using the FC approximation (thick black). The spectra are normalized so that the peak $n=1$ in $\sigma$ equals 1 . 


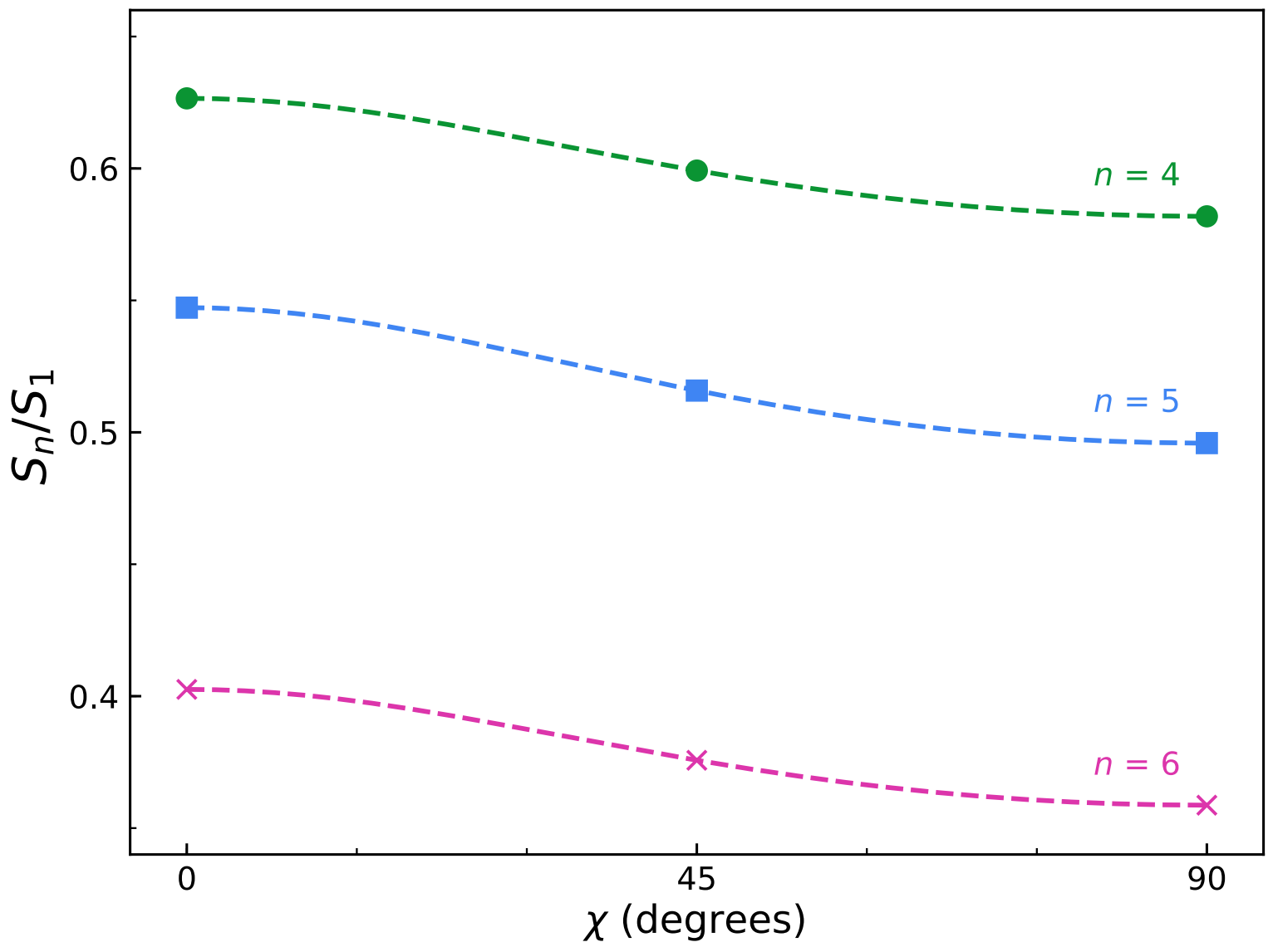

FIG. 6. Polarization dependence of the ratio of the areas of the $n$-th and the first peaks in the RIXS spectrum (see eq.(18)). 

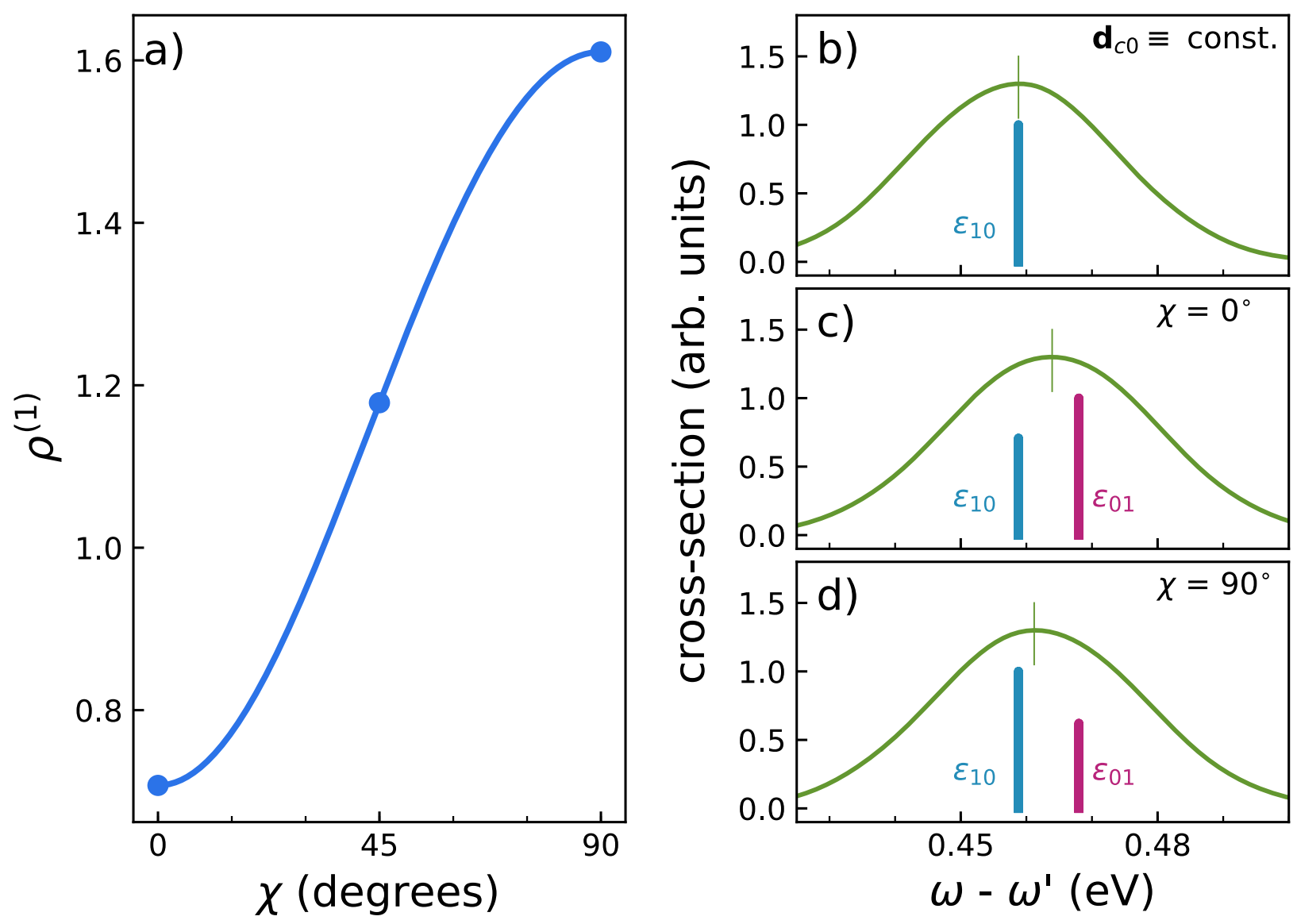

FIG. 7. a) Polarization dependence of the ratio $\rho^{(1)}$ of intensities of the symmetry allowed (10) and forbidden (01) RIXS channels (see eq.(19)). Panel b) displays $\sigma$ for the peak $n=1$ in the FC approximation. Panels c) and d) show the same taking into account the rotation of the transition dipole moment for $\chi=0^{\circ}$ and $90^{\circ}$, respectively. The total profile shown by thick green line is calculated using a Gaussian with half-width at half maximum equal to $0.02 \mathrm{eV}$. The RIXS cross-section bars for levels $\epsilon_{1,0}$ and $\epsilon_{0,1}$ are shown by thick blue and magenta bars, respectively. The vertical thin green lines show weak dependence of the peak position of unresolved peaks on the scattering angle $\chi$. 